February 11, 2020

\title{
KELLY CRITERION: FROM A SIMPLE RANDOM WALK TO LÉVY PROCESSES
}

\author{
SERGEY LOTOTSKY AND AUSTIN POLLOK
}

\begin{abstract}
The original Kelly criterion provides a strategy to maximize the longterm growth of winnings in a sequence of simple Bernoulli bets with an edge, that is, when the expected return on each bet is positive. The objective of this work is to consider more general models of returns and the continuous time, or high frequency, limits of those models.
\end{abstract}

\section{INTRODUCTION}

Consider repeatedly engaging in a game of chance where one side has an edge and seeks to optimize the betting in a way that ensures maximal long-term growth rate of the overall wealth. This problem was posed and analyzed by John Kelly [5] at Bell Labs in 1956; the solution was implemented and tested in a variety of setting by a successful mathematician, gambler, and hedge fund manager Ed Thorp [12] over the period from the 60 's to the early 00 's.

As a motivating example, consider betting on a biased coin toss where the return $r$ is a random variable with distribution

$$
\mathbb{P}(r=1)=p, \quad \mathbb{P}(r=-1)=1-p ;
$$

in what follows, we refer to this as the simple Bernoulli model. The condition to have an edge in this setting becomes $1 / 2<p \leq 1$ or, equivalently,

$$
\mathbb{E}[r]=2 p-1>0 .
$$

We plan on being able to make a large sequence of bets on this biased coin, resulting in an iid sequence of returns $\left\{r_{k}\right\}_{k \geq 1}$ with the same distribution as $r$, and ask how much we should bet so as to maximize long term wealth, given that we are compounding our returns. Assume we are betting with a fixed exposure $f$, that is, each bet involves a fixed fraction $f$ of the overall wealth, and $f \in[0,1]$. Practically, $f \geq 0$ means no shorting and $f \leq 1$ means no leverage, which we refer to as the NS-NL condition. Then, starting with the initial amount $W_{0}$, the total wealth at time $n=1,2,3, \ldots$ is the following function of $f$ :

$$
W_{n}^{f}=W_{0} \prod_{k=1}^{n}\left(1+f r_{k}\right)
$$

2010 Mathematics Subject Classification. Primary 60G50; Secondary 60F05, 60F17, 60G51, $91 \mathrm{~A} 60$.

Key words and phrases. Logarithmic Utility, Processes With Independent Increments, Weak Convergence. 
For the long-term compounder wishing to maximize their long term wealth, a natural and equivalent goal would be to find the strategy $f=f^{*}$ maximizing the long-term growth rate

$$
g_{r}(f):=\lim _{n \rightarrow \infty} \frac{1}{n} \ln \frac{W_{n}^{f}}{W_{0}}
$$

By direct computation,

$$
g_{r}(f)=\lim _{n \rightarrow \infty} \frac{1}{n} \sum_{k=1}^{n} \ln \left(1+f r_{k}\right)=\mathbb{E} \ln (1+f r)=p \ln (1+f)+(1-p) \ln (1-f),
$$

where the second equality follows by the law of large numbers, and therefore, after solving $g_{r}^{\prime}\left(f^{*}\right)=0$,

$$
f^{*}=2 p-1, \quad \max _{f \in[0,1]} g_{r}(f)=g_{r}\left(f^{*}\right)=p \ln \frac{p}{1-p}+(2-p) \ln (2-2 p) ;
$$

note that the edge condition (1.2) ensures that $f^{*}$ is an admissible strategy and $g_{r}\left(f^{*}\right)>0$. For more discussions of this result see [12].

Our objective in this paper is to derive analogues of (1.4) in the following situations:

(1) the distribution of returns is a more general random variable;

(2) the compounding is continuous in time;

(3) the compounding is high frequency, leading to a continuous-time limit.

In particular, we consider several scenarios when the returns are described by Lévy processes, which addresses some of Thorp's questions regarding fat-tailed distributions in finance [11].

In what follows, we write $\xi \stackrel{d}{=} \eta$ to indicate equality in distribution for two random variables, and $X \stackrel{\mathcal{L}}{=} Y$ to indicate equality in law (as function-valued random elements) for two random processes. For $x>0,\lfloor x\rfloor$ denotes the largest integer less than or equal to $x$. To simplify the notations, we always assume that $W_{0}=1$.

\section{Discrete Compounding: General Distribution of Returns}

Assume that the returns on each bet are independent random variables $r_{k}, k \geq 1$, with the same distribution as a given random variable $r$, and let

$$
W_{n}^{f}=\prod_{k=1}^{n}\left(1+f r_{k}\right)
$$

denote the corresponding wealth process. We also keep the NS-NL condition on admissible strategies: $f \in[0,1]$.

For the wealth process $W^{f}$ to be well-defined, we need the random variable $r$ to have the following properties:

$$
\begin{aligned}
& \mathbb{P}(r \geq-1)=1 \\
& \mathbb{P}(r>0)>0, \mathbb{P}(r<0)>0 \\
& \mathbb{E}|\ln (1+r)|<\infty
\end{aligned}
$$


Condition (2.2) quantifies the idea that a loss in a bet should not be more than 100\%. Condition (2.3) is basic non-degeneracy: both gains and losses are possible. Condition (2.4) is a minimal requirement to define the long-term growth rate of the wealth process.

The key object in this section will be the function

$$
g_{r}(f)=\mathbb{E} \ln (1+f r) .
$$

In particular, the following result shows that $g_{r}(f)$ is the long term growth rate of the wealth process $W^{f}$.

Proposition 2.1. If (2.2) and (2.4) hold and $g_{r}(f) \neq 0$, then, for every $f \in[0,1]$, the wealth process $W^{f}$ has an asymptotic representation

$$
W_{n}^{f}=\exp \left(n g_{r}(f)\left(1+\varepsilon_{n}\right)\right)
$$

where

$$
\lim _{n \rightarrow \infty} \varepsilon_{n}=0
$$

with probability one.

Proof. By (2.1), we have (2.6) with

$$
\varepsilon_{n}=\frac{1}{n g_{r}(f)} \sum_{k=1}^{n}\left(\ln \left(1+f r_{k}\right)-g_{r}(f)\right)
$$

and then (2.7) follows by (2.4) and the strong law of large numbers.

A stronger version of (2.4) leads to a more detailed asymptotic of $W_{n}^{f}$.

Theorem 2.1. Assume that (2.2) holds and

$$
\mathbb{E}|\ln (1+r)|^{2}<\infty .
$$

Then then, for every $f \in[0,1]$, the wealth process $W^{f}$ has an asymptotic representation

$$
W_{n}^{f}=\exp \left(n g_{r}(f)+\sqrt{n}\left(\sigma_{r}(f) \zeta_{n}+\epsilon_{n}\right)\right),
$$

where $\zeta_{n}, n \geq 1$, are standard Gaussian random variables,

$$
\sigma_{r}(f)=\left(\mathbb{E}\left[\ln ^{2}(1+f r)\right]-g_{r}^{2}(f)\right)^{1 / 2}
$$

and

$$
\lim _{n \rightarrow \infty} \epsilon_{n}=0
$$

in probability.

Proof. With $\varepsilon_{n}$ from (2.8), the result follows by the Central Limit Theorem:

$$
n g_{r}(f) \varepsilon_{n}=\sqrt{n}\left(\frac{1}{\sqrt{n}} \sum_{k=1}^{n}\left(\ln \left(1+f r_{k}\right)-g_{r}(f)\right)\right)=\sqrt{n}\left(\sigma_{r}(f) \zeta_{n}+\epsilon_{n}\right)
$$


Because the Central Limit Theorem gives convergence in distribution, the random variables $\zeta_{n}$ in (2.10) can indeed depend on $n$. Additional assumptions about the distribution of $r$ [14, Theorem 1] lead to higher-order asymptotic expansions and a possibility to have $\lim _{n \rightarrow \infty} \epsilon_{n}=0$ with probability one.

The following properties of the function $g_{r}$ are immediate consequences of the definition and the assumptions (2.2)-(2.4):

Proposition 2.2. The function $f \mapsto g_{r}(f)$ is continuous on the closed interval $[0,1]$ and infinitely differentiable in $(0,1)$. In particular,

$$
\frac{d g_{r}}{d f}(f)=\mathbb{E}\left[\frac{r}{1+f r}\right], \quad \frac{d^{2} g_{r}}{d f^{2}}(f)=-\mathbb{E}\left[\frac{r^{2}}{(1+f r)^{2}}\right]<0 .
$$

Corollary 2.1. The function $g_{r}$ achieves its maximal value on $[0,1]$ at a point $f^{*} \in$ $[0,1]$ and $g_{r}\left(f^{*}\right) \geq 0$. If $g_{r}\left(f^{*}\right)>0$, then $f^{*}$ is unique.

Proof. Note that $g_{r}(0)=0$ and, by (2.11), the function $g_{r}$ is strictly concave (or convex up) on $[0,1]$.

While concavity of $g_{r}$ implies that $g_{r}$ achieves a unique global maximal value at a point $f^{* *}$, it is possible that the domain of the function $g_{r}$ is bigger than the interval $[0,1]$ and $f^{* *} \notin[0,1]$. A simple way to exclude the possibility $f^{* *}<0$ is to consider returns $r$ that are not bounded from above: $\mathbb{P}(r>c)>0$ for all $c>0$ : in this case, the function $g_{r}(f)=\mathbb{E} \ln (1+f r)$ is not defined for $f<0$. Similarly, if $\mathbb{P}(r<-1+\delta)>0$ for all $\delta>0$, then the function $g_{r}$ is not defined for $f>1$, excluding the possibility $f^{* *}>1$.

Below are more general sufficient conditions to ensure that the point $f^{*} \in[0,1]$ from Corollary 2.1 is the point of global maximum of $g_{r}: f^{*}=f^{* *}$.

Proposition 2.3. If

$$
\begin{aligned}
& \lim _{f \rightarrow 0+} \mathbb{E}\left[\frac{r}{1+f r}\right]>0 \quad \text { and } \\
& \lim _{f \rightarrow 1-} \mathbb{E}\left[\frac{r}{1+f r}\right]<0,
\end{aligned}
$$

then there is a unique $f^{*} \in(0,1)$ such that

$$
g_{r}(f)<g_{r}\left(f^{*}\right)
$$

for all $f$ in the domain of $g_{r}$.

Proof. Together with the intermediate value theorem, conditions (2.12) and (2.13) imply that there is a unique $f^{*} \in(0,1)$ such that

$$
\frac{d g_{r}}{d f}\left(f^{*}\right)=0 .
$$

It remains to use strong concavity of $g_{r}$. 
Because $r \geq-1$, the expected value $\mathbb{E}[r]$ is always defined, although $\mathbb{E}[r]=+\infty$ is a possibility. Thus, by (2.11), condition (2.12) is equivalent to the intuitive idea of an edge:

$$
\mathbb{E}[r]>0,
$$

which, similar to (1.2), guarantees that $g_{r}(f)>0$ for some $f \in(0,1)$. Condition (2.13) can be written as

$$
\mathbb{E}\left[\frac{r}{1+r}\right]<0
$$

with the convention that the left-hand side can be $-\infty$. This condition does not appear in the simple Bernoulli model, but is necessary in general, to ensure that the edge is not too big and leveraged gambling $\left(f^{*}>1\right)$ does not lead to an optimal strategy.

As an example, consider the general Bernoulli model with

$$
\mathbb{P}(r=-a)=1-p, \quad \mathbb{P}(r=b)=p, \quad 0<a \leq 1, b>0,0<p<1 .
$$

The function

$$
g_{r}(f)=p \ln (1+f b)+(1-p) \ln (1-f a)
$$

is defined on $(-1 / b, 1 / a)$, achieves the global maximum at

$$
f^{*}=\frac{p}{a}-\frac{1-p}{b}
$$

and

$$
g_{r}\left(f^{*}\right)=p \ln p+(1-p) \ln (1-p)+\ln \frac{a+b}{a}-(p-1) \ln \frac{b}{a} ;
$$

we know that $g_{r}\left(f^{*}\right) \geq 0$, even though it is not at all obvious from the above expression.

The NS-NL condition $f^{*} \in[0,1]$ becomes

$$
\frac{a}{a+b} \leq p \leq \min \left(\frac{a b}{a+b}\left(1+\frac{1}{b}\right), 1\right)
$$

and it is now easy to come up with a model in which $f^{*}>1$ : for example, take

$$
a=0.1, b=0.5, p=0.5
$$

so that $f^{*}=4$. Given that a gain and a loss in each bet are equally likely, but the amount of a gain is five times as much as that of a loss, a large value of $f^{*}$ is not surprising, although economical and financial implications of this type of leveraged betting are potentially very interesting and should be a subject of a separate investigation.

Because of the logarithmic function in the definition of $g_{r}$, the distribution of $r$ can have a rather heavy right tail and still satisfy (2.4). For example, consider

$$
r=\eta^{2}-1
$$

where $\eta$ has standard Cauchy distribution with probability density function

$$
h_{\eta}(x)=\frac{1}{\pi\left(1+x^{2}\right)}, \quad-\infty<x<+\infty .
$$


Then

$$
g_{r}(f)=\frac{2}{\pi} \int_{0}^{+\infty} \frac{\ln \left((1-f)+f x^{2}\right)}{1+x^{2}} d x=2 \ln (\sqrt{f}+\sqrt{1-f}),
$$

where the second equality follows from [3, (4.295.7)]. As a result, we get a closed-form answer

$$
f^{*}=\frac{1}{2}, g_{r}\left(f^{*}\right)=\ln 2 .
$$

A general way to ensure $(2.2)-(2.4)$ is to consider

$$
r=e^{\xi}-1
$$

for some random variable $\xi$ such that $\mathbb{P}(\xi>0)>0, \mathbb{P}(\xi<0)>0$, and $\mathbb{E}|\xi|<\infty$; note that (2.15) is a particular case, with $\xi=\ln \eta^{2}$. Then (2.12) and (2.13) become, respectively,

$$
\begin{aligned}
& \mathbb{E} e^{\xi}>1 \quad \text { and } \\
& \mathbb{E} e^{-\xi}>1
\end{aligned}
$$

For example, if $\xi$ is normal with mean $\mu \in \mathbb{R}$ and variance $\sigma^{2}>0$, then

$$
\mathbb{E} e^{\xi}=e^{\mu+\left(\sigma^{2} / 2\right)}, \quad \mathbb{E} e^{-\xi}=e^{-\mu+\left(\sigma^{2} / 2\right)},
$$

and (2.17), (2.18) are equivalent to

$$
-\frac{\sigma^{2}}{2}<\mu<\frac{\sigma^{2}}{2}
$$

which, when interpreted in terms of returns, can indeed be considered as a "reasonable" edge condition: large values of $|\mu|$ do create a bias in one direction. Note that the corresponding $f^{*}$ is not available in closed form, but can be evaluated numerically.

\section{Continuous Compounding and a Case for Lévy Processes}

Continuous time compounding includes discrete compounding as a particular case and makes it possible to consider more general types of return processes. The objective of this section is to show that continuous time compounding that leads to a non-trivial and non-random long-term growth rate of the resulting wealth process effectively forces the return process to have independent increments. The two main examples of such process are sums of iid random variables from the previous section and the Lévy processes.

Writing (2.1) as

$$
W_{n+1}^{f}-W_{n}^{f}=\left(f W_{n}^{f}\right) r_{n+1},
$$

we see that a natural continuous time version of (3.1) is

$$
d W_{t}^{f}=f W_{t}^{f} d R_{t}
$$

for a suitable process $R=R_{t}, t \geq 0$ on a stochastic basis

$$
\mathbb{F}=\left(\Omega, \mathcal{F},\left\{\mathcal{F}_{t}\right\}_{t \geq 1}, \mathbb{P}\right)
$$


satisfying the usual conditions [9, Definition I.1.1]. We interpret (3.2) as an integral equation

$$
W_{t}^{f}=1+f \int_{0}^{t} W_{s}^{f} d R_{s}
$$

recall that $W_{0}^{f}=1$ is the standing assumption. Then the Bichteler-Dellacherie theorem [9, Theorem III.22] implies that the process $R$ must be a semi-martingale (a sum of a martingale and a process of bounded variation) with trajectories that, at every point, are continuous from the right and have limits from the left. Furthermore, if we allow the process $R$ to have discontinuities, then, by [9, Theorem II.36], we need to modify (3.3) further:

where

$$
W_{t}^{f}=1+f \int_{0}^{t} W_{s-}^{f} d R_{s}
$$

$$
W_{s-}=\lim _{\varepsilon \rightarrow 0, \varepsilon>0} W_{s-\varepsilon},
$$

and, assuming $R_{0}=0$, the process $W^{f}$ becomes the Doléans-Dade exponential

$$
W_{t}^{f}=\exp \left(f R_{t}-\frac{f^{2}\left\langle R^{c}\right\rangle_{t}}{2}\right) \prod_{0<s \leq t}\left(1+f \triangle R_{s}\right) e^{-f \triangle R_{s}} .
$$

In (3.4),$\left\langle R^{c}\right\rangle$ is the quadratic variation process of the continuous martingale component of $R$ and $\triangle R_{s}=R_{s}-R_{s-}$.

A natural analog of (2.2) is

$$
\triangle R_{s} \geq-1
$$

and then (3.4) becomes

$$
W_{t}^{f}=\exp \left(f R_{t}-\frac{f^{2}\left\langle R^{c}\right\rangle_{t}}{2}+\sum_{0<s \leq t}\left(\ln \left(1+f \triangle R_{s}\right)-f \triangle R_{s}\right)\right) .
$$

To proceed, let us assume that the trajectories of $R$ are continuous: $\triangle R_{s}=0$ for all $s$ so that

$$
W_{t}^{f}=\exp \left(f R_{t}-f^{2}\left\langle R^{c}\right\rangle_{t}\right)
$$

If, similar to (1.3), we define the long-term growth rate $g_{R}(f)$ by

$$
g_{R}(f)=\lim _{t \rightarrow \infty} \frac{\ln W_{t}^{f}}{t},
$$

then we need the limits

$$
\mu:=\lim _{t \rightarrow \infty} \frac{R_{t}}{t}, \quad \sigma^{2}:=\lim _{t \rightarrow \infty} \frac{\left\langle R^{c}\right\rangle_{t}}{t}
$$

to exist with probability one and with non-random numbers $\mu, \sigma^{2}$. Being a semimartingale without jumps, the process $R$ has a representation

$$
R_{t}=A_{t}+R_{t}^{c}
$$

where $A$ is process of bounded variation; cf. [4, Theorem II.2.34]. Then (3.8) imply that, for large $t$,

$$
A_{t} \approx \mu t,\left\langle R^{c}\right\rangle_{t} \approx \sigma^{2} t
$$


that is, a natural way to achieve (3.8) is to consider the process $R$ of the form

$$
R_{t}=\mu t+\sigma B_{t}
$$

where $\sigma>0$ and $B=B_{t}$ is a standard Brownian motion. Then

$$
W_{t}^{f}=\exp \left(f \mu t+f \sigma B_{t}-\frac{f^{2} \sigma^{2} t}{2}\right)
$$

and we come to the following conclusion: continuous time compounding with a continuous return process effectively implies that the wealth process is a geometric Brownian motion.

The long-term growth rate (3.7) becomes

$$
g_{R}(f)=f \mu-\frac{f^{2} \sigma^{2}}{2},
$$

so that

$$
f^{*}=\frac{\mu}{\sigma^{2}}, \quad g_{R}\left(f^{*}\right)=\frac{\mu^{2}}{2 \sigma^{2}},
$$

and the NS-NL condition is

$$
0<\mu<\sigma^{2}
$$

Even though these results are not especially sophisticated, we will see in the next section (Theorem 4.1) that the process (3.11) naturally appears as the continuoustime, or high frequency, limit of discrete-time compounding for a large class of returns.

On the other hand, if we assume that the process $R$ is purely discontinuous, with jumps $\triangle R_{k}=r_{k}$ at times $s=k \in\{1,2,3, \ldots\}$, then

$$
R_{t}=0, t \in(0,1), R_{t}=\sum_{k=1}^{\lfloor t\rfloor} r_{k}, t \geq 1
$$

and (3.4) becomes (2.1). Accordingly, we will now investigate the general case (3.4) when the process $R$ has both a continuous component and jumps. To this end, we use [4, Proposition II.1.16] and introduce the jump measure $\mu^{R}=\mu^{R}(d x, d s)$ of the process $R$ by putting a point mass at every point in space-time where the process $R$ has a jump:

$$
\mu^{R}(d x, d s)=\sum_{s>0} \delta_{\left(\triangle R_{s}, s\right)}(d x, d s)
$$

note that both the time $s$ and size $\triangle R_{s}$ of the jump can be random. In particular, with (3.5) in mind,

$$
\sum_{0<s \leq t}\left(\ln \left(1+f \triangle R_{s}\right)-f \triangle R_{s}\right)=\int_{0}^{t} f_{-1}^{+\infty}(\ln (1+f x)-f x) \mu^{R}(d x, d s) ;
$$

here and below,

stands for

$$
f_{a}^{b}, \quad a<0<b
$$

$$
\int_{(a, 0) \cup(0, b)}
$$


By [4, Proposition II.2.9 and Theorem II.2.34], and keeping in mind (3.5), we get the following generalization of (3.9):

$$
\begin{aligned}
R_{t} & =A_{t}+R_{t}^{c}+\int_{0}^{t} f_{1}^{+\infty} x \mu^{R}(d x, d s) \\
& +\int_{0}^{t} f_{-1}^{1} x\left(\mu^{R}(d x, d s)-\nu(d x, s) d a_{s}\right),
\end{aligned}
$$

where $a=a_{t}$ is a predictable non-decreasing process and $\nu=\nu(d x, t)$ is the nonnegative random time-dependent measure on $(-1,0) \bigcup(0,+\infty)$ with the property

$$
f_{-1}^{+\infty} \min \left(1, x^{2}\right) \nu(d x, t) \leq 1
$$

for all $t \geq 0$ and $\omega \in \Omega$. Moreover

$$
\begin{aligned}
A_{t} & =\int_{0}^{t} \mu_{s} d a_{s} \text { for some predictable process } \mu=\mu_{t}, \\
\left\langle R^{c}\right\rangle_{t} & =\int_{0}^{t} \sigma_{s}^{2} d a_{s} \text { for some predictable process } \sigma=\sigma_{t},
\end{aligned}
$$

and the process

$$
t \mapsto \int_{0}^{t} f_{-1}^{+\infty} h(x)\left(\mu^{R}(d x, d s)-\nu(d x, s) d a_{s}\right)
$$

is a martingale for every bounded measurable function $h=h(x)$ such that $\lim \sup _{x \rightarrow 0}|h(x)| /|x|<\infty$.

To proceed, we assume that

$$
\mathbb{E} \int_{0}^{t} \int_{-1}^{+\infty}|\ln (1+x)| \nu(d x, s) d a_{s}<\infty, t>0,
$$

which is a generalization of condition (2.4). Then, by [4, Theorem II.1.8], the process

$$
t \mapsto \int_{0}^{t} \int_{-1}^{+\infty} \ln (1+x)\left(\mu^{R}(d x, d s)-\nu(d x, s) d a_{s}\right)
$$

is a martingale.

Next, we combine (3.6), (3.14), and (3.16), and re-arrange the terms so that the logarithm of the wealth process becomes

$$
\begin{aligned}
\ln W_{t}^{f} & =f A_{t}+f R_{t}^{c}-\frac{f^{2}}{2}\left\langle R_{t}^{c}\right\rangle-f \int_{0}^{t} f_{-1}^{1} x \nu(d x, s) d a_{s} \\
& +\int_{0}^{t} f_{-1}^{+\infty} \ln (1+f x) \nu(d x, s) d a_{s}+M_{t}^{f},
\end{aligned}
$$

where

$$
M_{t}^{f}=\int_{0}^{t} f_{-1}^{+\infty} \ln (1+f x)\left(\mu^{R}(d x, d s)-\nu(d x, s) d a_{s}\right) .
$$


In general, for equality (3.19) to hold, we need to make an additional assumption

$$
\int_{-1}^{1} x \nu(d x, t)<\infty
$$

for all $t \geq 0$ and $\omega \in \Omega$.

In the particular case (2.1),

- $a_{s}=\lfloor s\rfloor$ is the step function, with unit jumps at positive integers, so that $d a_{s}$ is the collection of point masses at positive integers;

- $\nu(d x, s)=F^{R}(d x)$, where $F^{R}$ is the cumulative distribution function of the random variable $r$, so that (3.20) holds automatically;

- $\mu_{t}=g_{f}(r)+\int_{-1}^{1} x F^{R}(d x), R_{t}^{c}=0, \sigma_{t}=0$;

- $M_{t}^{f}=\sum_{0<k \leq t}\left(\ln \left(1+f r_{k}\right)-g_{r}(f)\right)$;

- condition (3.25) is (2.9).

A natural way to reconcile (3.10) with (3.17), (3.18) is to take $\mu_{t}=\mu, \sigma_{t}=\sigma$ for some non-random numbers $\mu \in \mathbb{R}, \sigma \geq 0$, and a non-random non-decreasing function $a=a_{t}$ with the property

$$
\lim _{t \rightarrow+\infty} \frac{a_{t}}{t}=1
$$

Then, to have a non-random almost-sure limit

$$
\lim _{t \rightarrow \infty} \frac{1}{t} \int_{0}^{t} \int_{-1}^{+\infty} \varphi(x) \nu(d x, s) d a_{s}
$$

for a sufficiently rich class of non-random test functions $\varphi$, we have to assume that there exists a non-random non-negative measure $F^{R}=F^{R}(d x)$ on $(-1,0) \bigcup(0,+\infty)$ such that

$$
f_{-1}^{+\infty} \min (|x|, 1) F^{R}(d x)<\infty
$$

and, for large $s$,

$$
\nu(d x, s) \approx F^{R}(d x)
$$

As a result, if

$$
\nu(d x, s)=F^{R}(d x)
$$

for all $s$, then

$$
A_{t}=\mu a_{t},\left\langle R^{c}\right\rangle_{t}=\sigma^{2} a_{t}, \nu(d x, t)=F^{R}(d x) a_{t}
$$

are all non-random, and [4, Theorem II.4.15] implies that $R$ is a process with independent increments. Furthermore, (3.24) and the strong law of large numbers for martingales imply

$$
\mathbb{P}\left(\lim _{t \rightarrow \infty} \frac{R_{t}^{c}}{t}=0\right)=1 ;
$$

cf. [8, Corollary 1 to Theorem II.6.10]. Similarly, if

$$
\int_{-1}^{+\infty} \ln ^{2}(1+x) F^{R}(d x)<\infty
$$


then $M^{f}$ is a square-integrable martingale and

$$
\mathbb{P}\left(\lim _{t \rightarrow \infty} \frac{M_{t}^{f}}{t}=0\right)=1
$$

Writing

$$
\bar{\mu}=\mu-\int_{-1}^{1} x F^{R}(d x)
$$

the long-term growth rate (3.7) becomes

$$
g_{R}(f)=f \bar{\mu}-\frac{f^{2} \sigma^{2}}{2}+f_{-1}^{\infty} \ln (1+f x) F^{R}(d x),
$$

which does include both (2.5) and (3.12) as particular cases. By direct computation, the function $f \mapsto g_{R}(f)$ is concave and the domain of the function contains $[0,1]$.

Similar to Proposition 2.3, we have the following result.

Theorem 3.1. Consider continuous-time compounding with return process

$$
\begin{aligned}
R_{t} & =A_{t}+R_{t}^{c}+\int_{0}^{t} f_{1}^{+\infty} x \mu^{R}(d x, d s) \\
& +\int_{0}^{t} f_{-1}^{1} x\left(\mu^{R}(d x, d s)-\nu(d x, s) d a_{s}\right),
\end{aligned}
$$

where the random measure $\mu^{R}$ is from (3.13), and assume that equalities (3.21) and (3.24) hold. If $F^{R}$ satisfies (3.22), (3.25), and

$$
\begin{aligned}
& \lim _{f \rightarrow 0+} \int_{-1}^{\infty} \frac{x}{1+f x} F^{R}(d x)>-\bar{\mu} \\
& \lim _{f \rightarrow 1-} \int_{-1}^{\infty} \frac{x}{1+f x} F^{R}(d x)<\sigma^{2}-\bar{\mu}
\end{aligned}
$$

then the long-term growth rate is given by (3.26), and there exists a unique $f^{*} \in(0,1)$ such that

$$
g_{R}(f)<g_{R}\left(f^{*}\right)
$$

for all $f$ in the domain of $g_{R}$.

By the Lebesgue decomposition theorem, the measure corresponding to the function $a=a_{t}$ has a discrete, absolutely continuous, and singular components. With (3.21) in mind, a natural choice of the discrete component is $a_{t}=\lfloor t\rfloor$, which, as we saw, corresponds to discrete compounding discussed in the previous section. A natural choice of the absolutely continuous component is

$$
a_{t}=t
$$

Then

$$
A_{t}=\mu t, R_{t}^{c}=\sigma B_{t}, \nu(d x, t) d a_{t}=F^{R}(d x) d t,
$$

where $B$ is a standard Brownian motion. By [4, Corollary II.4.19], we conclude that the process $R$ has independent and stationary increments, that is, $R$ is a Lévy process. 
In this case, equality (3.27) is known as the Lévy-Itô decomposition of the process $R$; cf. [10, Theorem 19.2].

We do not consider the singular case in this paper and leave it for future investigation.

\section{Continuous Limit of Discrete Compounding}

4.1. A (Simple) Random Walk Model. Following the methodology in [12, Section 7.1], we assume compounding a sufficiently large number $n$ of bets in a time period $[0, T]$. The returns $r_{n, 1}, r_{n, 2}, \ldots$ of the bets are

$$
r_{n, k}=\frac{\mu}{n}+\frac{\sigma}{\sqrt{n}} \xi_{n, k}
$$

for some $\mu>0, \sigma>0$ and independent identically distributed random variables $\xi_{n, k}, k=1,2, \ldots$, with mean 0 and variance 1 . The classical simple random walk corresponds to $\mathbb{P}\left(\xi_{n, k}= \pm 1\right)=1 / 2$ and can be considered a high frequency version of (1.1). Similar to (2.2), we need $r_{n, k} \geq-1$, which, in general, can only be achieved with uniform boundedness of $\xi_{n, k}$ :

$$
\left|\xi_{n, k}\right| \leq C_{0}
$$

and then, with no loss of generality, we assume that $n$ is large enough so that

$$
\left|r_{n, k}\right| \leq \frac{1}{2}
$$

Similar to (1.2), a condition to have an edge is

$$
\mathbb{E}\left[r_{n, k}\right]=\frac{\mu}{n}>0
$$

and, similar to (2.1), given $n$ bets per unit time period, with exposure $f \in[0,1]$ in each bet, we get the following formula for the total wealth $W_{t}^{n, f}$ at time $t \in(0, T]$ assuming $W_{0}=1$ :

$$
W_{t}^{n, f}=\prod_{k=1}^{\lfloor n t\rfloor}\left(1+f r_{n, k}\right)
$$

$\lfloor n t\rfloor$ denotes the largest integer less than $n t$.

Let $B=B_{t}, t \geq 0$, be a standard Brownian motion on a stochastic basis $\left(\Omega, \mathcal{F},\left\{\mathcal{F}_{t}\right\}_{t \geq 0}, \mathbb{P}\right)$ satisfying the usual conditions, and define the process

$$
W_{t}^{f}=\exp \left(\left(f \mu-\frac{f^{2} \sigma^{2}}{2}\right) t+f \sigma B_{t}\right) .
$$

Note that (4.5) is a particular case of (3.11).

Theorem 4.1. For every $T>0$ and every $f \in[0,1]$, the sequence of processes $\left(W_{t}^{n, f}, n \geq 1, t \in[0, T]\right)$ converges in law to the process $W^{f}=W_{t}^{f}, t \in[0, T]$.

Proof. Writing

$$
Y_{t}^{n, f}=\ln W_{t}^{n, f}
$$


the objective is to show weak convergence, as $n \rightarrow \infty$, of $Y^{n, f}$ to the process

$$
Y_{t}^{f}=\left(f \mu-\frac{f^{2} \sigma^{2}}{2}\right) t+f \sigma B_{t}, \quad t \in[0, T] .
$$

The proof relies on the method of predictable characteristics for semimartingales from [4]. More specifically, we make suitable changes in the proof of Corollary VII.3.11.

By 4.5

$$
Y_{t}^{n, f}=\sum_{k=1}^{\lfloor n t\rfloor} \ln \left(1+f r_{n, k}\right)
$$

Then (4.1) and (4.2) imply

$$
\mathbb{E}\left(Y_{t}^{n, f}-\mathbb{E} Y_{t}^{n, f}\right)^{4} \leq \frac{C_{0}^{4} \sigma^{4}}{n^{2}}(n T+3 n T(n T-1)) \leq 3 C_{0}^{4} \sigma^{4} T^{2},
$$

from which uniform integrability of the family $\left\{Y_{t}^{n, f}, n \geq 1, t \in[0, T]\right\}$ follows.

Then, by [4, Theorem VII.3.7], it suffices to establish the following:

$$
\begin{aligned}
& \lim _{n \rightarrow \infty} \sup _{t \leq T}\left\lfloor\lfloor n t\rfloor \mathbb{E}\left[\ln \left(1+f r_{n, 1}\right)\right]-\left(f \mu-\frac{f^{2} \sigma^{2}}{2}\right) t \mid=0,\right. \\
& \lim _{n \rightarrow \infty}\lfloor n t\rfloor\left(\mathbb{E}\left(\ln \left(1+f r_{n, 1}\right)\right)^{2}-\left(\mathbb{E}\left[\ln \left(1+f r_{n, 1}\right)\right]\right)^{2}\right)=f^{2} \sigma^{2} t, t \in[0, T], \\
& \lim _{n \rightarrow \infty}\lfloor n t\rfloor \mathbb{E}\left[\phi\left(\ln \left(1+f r_{n, 1}\right)\right)\right]=0, t \in[0, T] .
\end{aligned}
$$

Equality (4.8) must hold for all functions $\phi=\phi(x), x \in \mathbb{R}$, that are continuous and bounded on $\mathbb{R}$ and satisfy $\phi(x)=o\left(x^{2}\right), x \rightarrow 0$, that is,

$$
\lim _{x \rightarrow 0} \frac{\phi(x)}{x^{2}}=0
$$

Equalities (4.6) and (4.7) follow from

$$
r_{n, 1}^{2}=\frac{\sigma^{2}}{n} \xi_{n, 1}^{2}+\frac{2 \mu \sigma \xi_{n, 1}}{n^{3 / 2}}+\frac{\mu^{2}}{n^{2}},
$$

together with (4.3) and an elementary inequality

$$
\left|\ln (1+x)-x-\frac{x^{2}}{2}\right| \leq|x|^{3},|x| \leq \frac{1}{2} .
$$

In particular,

$$
\mathbb{E}\left[\left(\ln \left(1+f r_{n, 1}\right)\right)^{2}\right]=\frac{f^{2} \sigma^{2}}{n}+o(1 / n), n \rightarrow+\infty .
$$

To establish (4.8), note that (4.9) and (4.1) imply

$$
\phi\left(\ln \left(1+f r_{n, 1}\right)\right)=o(1 / n), n \rightarrow+\infty .
$$


Similar to (1.3), we define the long-term continuous time growth rate

$$
g(f)=\lim _{t \rightarrow \infty} \frac{1}{t} \ln W_{t}^{f} .
$$

Then a simple computation show that

$$
g(f)=f \mu-\frac{f^{2} \sigma^{2}}{2}
$$

and so

$$
f^{*}=\frac{\mu}{\sigma^{2}}
$$

achieves the maximal long-term continuous time growth rate

$$
g\left(f^{*}\right)=\frac{\mu^{2}}{2 \sigma^{2}}
$$

The NS-NL condition $f^{*} \in[0,1]$ holds if $0 \leq \mu \leq \sigma^{2}$, which, to the order $1 / n$, is consistent with (2.12) and (2.13), when applied to (4.1):

$$
\mathbb{E}\left[r_{n, k}\right]=\frac{\mu}{n}, \quad \mathbb{E}\left[\frac{r_{n, k}}{1+r_{n, k}}\right]=\frac{\mu-\sigma^{2}}{n}+o\left(n^{-1}\right) .
$$

The wealth process (4.5) is that of someone who is "continuously" placing bets, that is, adjusts the positions instantaneously, and, for large $n$, is a good approximation of high frequency betting (4.4). In general, when the returns are given by (4.1), a direct optimization of (4.4) with respect to $f$ will not lead to a closed-form expression for the corresponding optimal strategy $f_{n}^{*}$, but Theorem 4.1 implies that, for sufficiently large $n$, (4.10) is an approximation of $f_{n}^{*}$ and (4.11) is an approximation of the corresponding long-term growth rate. As an illustration, consider the high-frequency version of the simple Bernoulli model (1.1):

$$
\mathbb{P}\left(r_{n, k}=\frac{\mu}{n} \pm \frac{\sigma}{\sqrt{n}}\right)=\frac{1}{2}
$$

which, for fixed $n$, is is a particular case of the general Bernoulli model (2.14) with $p=q=1 / 2$,

$$
a=\frac{\sigma}{\sqrt{n}}-\frac{\mu}{n}, b=\frac{\sigma}{\sqrt{n}}+\frac{\mu}{n}
$$

Then, by direct computation,

$$
f_{n}^{*}=\frac{\mu}{\sigma^{2}-\left(\mu^{2} / n\right)} \rightarrow \frac{\mu}{\sigma^{2}}, n \rightarrow \infty,
$$

and

$$
\lim _{n \rightarrow \infty} g_{r_{n}}\left(f_{n}^{*}\right)=\frac{\mu^{2}}{2 \sigma^{2}} .
$$

Even though the analysis of the proof of Theorem 4.1 shows that the convergence is uniform in $f$ on compact sub-sets of $(0,1)$, the proof that $\lim _{n \rightarrow \infty} f_{n}^{*}=f^{*}$ would require a version of Theorem 4.1 with $T=+\infty$, which, for now, is not available.

With natural modifications, Theorem 4.1 extends to the setting (2.16) . 
Theorem 4.2. Assume that

$$
r_{n, k}+1=\exp \left(\frac{b}{n}+\frac{\sigma}{\sqrt{n}} \xi_{n, k}\right),
$$

where $b \in \mathbb{R}, \sigma>0$, and, for each $n \geq 1, k \leq n$, the random variables $\xi_{n, k}$ are independent and identically distributed, with zero mean, unit variance, and, for every $a>0$,

$$
\lim _{n \rightarrow \infty} n \mathbb{E}\left[\left|\xi_{n, 1}\right|^{2} I\left(\left|\xi_{n, 1}\right|>a \sqrt{n}\right)\right]=0 .
$$

Then the conclusion of Theorem 4.1 holds with

$$
\mu=b+\frac{\sigma^{2}}{2} \text {. }
$$

Proof. Even though a formal Taylor expansion suggests

$$
r_{n, k}=\frac{\mu}{n}+\frac{\sigma}{\sqrt{n}} \xi_{n, k}+o(1 / n)
$$

we cannot apply Theorem 4.1 directly because the random variables $\xi_{n, k}$ are not necessarily uniformly bounded. Still, condition (4.13) makes it possible to verify conditions (4.6)-(4.8).

Condition (4.13) is clearly satisfied when $\xi_{n, k}, k=1,2, \ldots$, are iid standard normal, which corresponds to

$$
r_{n, k}=\frac{P_{k / n}-P_{(k-1) / n}}{P_{(k-1) / n}}
$$

and

$$
P_{t}=e^{b t+\sigma B_{t}} .
$$

Thus, while the exponential model (2.16) with log-normal returns is not solvable in closed form, the high-frequency version leads to the (approximately) optimal strategy

$$
f^{*}=\frac{b}{\sigma^{2}}+\frac{1}{2}
$$

and, under (2.19), the NS-NL condition holds: $f^{*} \in(0,1)$. Numerical experiments with $\sigma=1$ and $n=10$ show that the values of the corresponding optimal $f_{10}^{*}$ are very close to those given by (4.16) for all $b \in(-1 / 2,1 / 2)$.

Informally, both Theorems 4.1 and 4.2 can be considered as particular cases of the delta method for the Donsker theorem with drift: if the sequence of processes

$$
t \mapsto \sum_{k=1}^{\lfloor n t\rfloor} \xi_{n, k}
$$

converges, as $n \rightarrow \infty$, to the processes $t \mapsto b t+\sigma B_{t}$ and $\varphi=\varphi(x)$ is a suitable function with $\varphi(0)=0$, then one would expect the sequence of processes

$$
t \mapsto \sum_{k=1}^{\lfloor n t\rfloor} \varphi\left(\xi_{n, k}\right)
$$


to converge to the process

$$
t \mapsto\left(\varphi^{\prime}(0) b+\frac{\varphi^{\prime \prime}(0) \sigma^{2}}{2}\right) t+\left|\varphi^{\prime}(0)\right| \sigma B_{t}
$$

4.2. Beyond the Log-Normal Limit. With the results of Section 3 in mind, we consider the following generalization of (4.14): (4.15):

$$
r_{n, k}=\frac{P_{k / n}-P_{(k-1) / n}}{P_{(k-1) / n}}, k=1,2, \ldots,
$$

where the process $P=P_{t}, t \geq 0$, has the form $P_{t}=e^{R_{t}}$, and $R=R_{t}$ is a Lévy process. In other words,

$$
r_{n, k}=e^{R_{k / n}-R_{(k-1) / n}}-1 .
$$

As in (3.27), the process $R=R_{t}$ can be decomposed into a drift, diffusion/small jump, and large jump components according to the Lévy-Itô decomposition [10, Theorem 19.2]:

$$
R_{t}=\mu t+\sigma B_{t}+\int_{0}^{t} f_{-1}^{1} x\left(\mu^{R}(d x, d s)-F^{R}(d x) d s\right)+\int_{0}^{t} \int_{|x|>1} x \mu^{R}(d x, d s)
$$

we continue to use the notation $f$ first introduced in (3.15).

Now that the process $R_{t}$ is exponentiated,

- there is no need to assume that $\triangle R_{t} \geq-1$;

- the analog of (3.25) becomes $\mathbb{E}\left|R_{1}\right|<\infty$.

Equality (4.18) has a natural interpretation in terms of financial risks [13]: the drift represents the edge ("guaranteed" return), diffusion and small jumps represent small fluctuations of returns, and the large jump component represents (sudden) large changes in returns. Similar to (4.4), the corresponding wealth process is

$$
W_{t}^{n, f}=\prod_{k=1}^{\lfloor n t\rfloor}\left(1+f r_{n, k}\right)
$$

We have the following generalization of Theorem 4.2.

Theorem 4.3. Consider the family of processes $W^{n, f}=W_{t}^{n, f}, t \in[0, T], n \geq 1$, $f \in[0,1]$, defined by (4.19). If $r_{n, k}$ is given by (4.17), with $P_{t}=e^{R_{t}}$, and $R=R_{t}$ is a Lévy process with representation (4.18) and $\mathbb{E}\left|R_{1}\right|<\infty$, then, for every $f \in[0,1]$ and $T>0$,

$$
\lim _{n \rightarrow \infty} W^{n, f} \stackrel{\mathcal{L}}{=} W^{f}
$$

in $\mathbb{D}((0, T))$, where

$$
\begin{aligned}
W_{t}^{f} & =\exp \left(f R_{t}+\frac{f(1-f) \sigma^{2}}{2} t\right. \\
& \left.+\int_{0}^{t} f_{\mathbb{R}}\left[\ln \left(1+f\left(e^{x}-1\right)\right)-f x\right] \mu^{R}(d x, d s)\right) .
\end{aligned}
$$


Proof. By (4.17) and (4.19),

$$
\ln W_{t}^{n, f}=\sum_{k=1}^{\lfloor n t\rfloor} \ln \left(1+f\left(e^{R_{k / n}-R_{(k-1) / n}}-1\right)\right) .
$$

Step 1: For $s \in\left(\frac{k-1}{n}, \frac{k}{n}\right]$, let

$$
r_{s}^{n, k}=e^{R_{s}-R_{(k-1) / n}}-1,
$$

and apply the Itô's formula [9, Theorem II.32] to the process

$$
s \mapsto \ln \left(1+f r_{s}^{n, k}\right), \quad s \in\left(\frac{k-1}{n}, \frac{k}{n}\right] .
$$

The result is

$$
\begin{aligned}
\ln \left(1+f r_{s}^{n, k}\right) & =\int_{\frac{k-1}{n}}^{s} \frac{f\left(1+r_{u-}^{n, k}\right)}{1+f r_{u-}^{n, k}} d R_{u}+\frac{\sigma^{2}}{2} \int_{\frac{k-1}{n}}^{s} \frac{f(1-f)\left(1+r_{u-}^{n, k}\right)}{\left(1+f r_{u-}^{n, k}\right)^{2}} d u \\
& +\int_{\frac{k-1}{n}}^{s} f_{\mathbb{R}}\left[\ln \left(1-f+f e^{x}\left(r_{u-}^{n, k}+1\right)\right)\right. \\
& \left.-\ln \left(1+f r_{u-}^{n, k}\right)-x \frac{f\left(1+r_{u-}^{n, k}\right)}{1+f r_{u-}^{n, k}}\right] \mu^{R}(d x, d u) .
\end{aligned}
$$

Step 2: Putting $s=\frac{k}{n}$ in the above equality and summing over $k$, we derive the following expression for $\ln W_{t}^{n, f}$ :

$$
\begin{aligned}
\ln W_{t}^{n, f} & =\sum_{k=1}^{\lfloor n t\rfloor}\left(\int_{\frac{k-1}{n}}^{\frac{k}{n}} h_{n, k}^{(1)}(s) d R_{s}+\int_{\frac{k-1}{n}}^{\frac{k}{n}} h_{n, k}^{(2)}(s) d s+\int_{\frac{k-1}{n}}^{\frac{k}{n}} f_{\mathbb{R}} h_{n, k}^{(3)}(s, x) \mu^{R}(d x, d u)\right) \\
& =\int_{0}^{t} H_{n, t}^{(1)}(s) d R_{s}+\int_{0}^{t} H_{n, t}^{(2)}(s) d s+\int_{0}^{t} f_{\mathbb{R}} H_{n, t}^{(3)}(s, x) \mu^{R}(d x, d s),
\end{aligned}
$$

where

$$
\begin{aligned}
h_{n, k}^{(1)}(s) & =\frac{f\left(1+r_{s-}^{n, k}\right)}{1+f r_{s-}^{n, k}}, \quad h_{n, k}^{(2)}(s)=\frac{\sigma f(1-f)}{2} \frac{1+r_{s-}^{n, k}}{\left(1+f r_{s-}^{n, k}\right)^{2}}, \\
h_{n, k}^{(3)}(s, x) & =\ln \left(1-f+f e^{x}\left(r_{s-}^{n, k}+1\right)\right)-\ln \left(1+f r_{s-}^{n, k}\right)-f x \frac{1+r_{s-}^{n, k}}{1+f r_{s-}^{n, k}} ; \\
H_{n, t}^{(i)}(s) & =\sum_{k=1}^{\lfloor n t\rfloor} h_{n, k}^{(i)}(s) \mathbf{1}_{\left(\frac{k-1}{n}, \frac{k}{n}\right]}(s), i=1,2 ; \quad H_{n, t}^{(3)}(s, x)=\sum_{k=1}^{\lfloor n t\rfloor} h_{n, k}^{(3)}(s, x) \mathbf{1}_{\left(\frac{k-1}{n}, \frac{k}{n}\right]}(s) .
\end{aligned}
$$

Step 3: Because

$$
\lim _{n \rightarrow \infty, k / n \rightarrow s} R_{(k-1) / n}=R_{s-}
$$

equality (4.21) implies

$$
\lim _{n \rightarrow+\infty, k / n \rightarrow s} r_{s-}^{n, k}=0
$$


for all $s$. Consequently, we have the following convergence in probability:

$$
\begin{aligned}
\lim _{n \rightarrow+\infty} H_{n, t}^{(1)}(s)=f, & \lim _{n \rightarrow+\infty} H_{n, t}^{(2)}(s)=\frac{\sigma^{2} f(1-f)}{2}, \\
& \lim _{n \rightarrow+\infty} H_{n, t}^{(2)}(s, x)=\ln \left(1+f\left(e^{x}-1\right)\right)-f x .
\end{aligned}
$$

To pass to the corresponding limits in (4.22), we need suitable bounds on the functions $H^{(i)}, i=1,2,3$.

Using the inequalities

$$
0<\frac{1+y}{1+a y} \leq \frac{1}{a}, \quad 0<\frac{1+y}{(1+a y)^{2}} \leq \frac{1}{4 a(1-a)}, \quad y>-1, a \in(0,1),
$$

we conclude that

$$
0<h_{n, k}^{(1)}(s) \leq 1,0<h_{n, k}^{(2)}(s) \leq \sigma^{2}
$$

and therefore

$$
0<H_{n, t}^{(1)}(s) \leq 1,0<H_{n, t}^{(2)}(s) \leq \sigma^{2} .
$$

Similarly, for $f \in(0,1)$ and $y>-1$,

$$
\left|\ln \frac{1-f+f e^{x}(y+1)}{1+f y}-f x \frac{1+y}{1+f y}\right| \leq 2\left(|x| \wedge|x|^{2}\right),
$$

so that

and

$$
\left|h_{n, k}^{(3)}(s, x)\right| \leq 2\left(|x| \wedge|x|^{2}\right)
$$

$$
\left|H_{n, t}^{(3)}(s)\right| \leq 2\left(|x| \wedge|x|^{2}\right) .
$$

To verify (4.24), fix $f \in(0,1)$ and $y>-1$, and define the function

$$
z(x)=\ln \frac{1-f+f e^{x}(y+1)}{1+f y}, x \in \mathbb{R} .
$$

By direct computation,

$$
\begin{aligned}
z(0) & =0, \\
z^{\prime}(x) & =\frac{f e^{x}(y+1)}{1-f+f e^{x}(y+1)}=1-\frac{1-f}{1-f+f e^{x}(y+1)}, \\
z^{\prime}(0) & =\frac{f(y+1)}{1+f y},
\end{aligned}
$$

so that, using the Taylor formula,

$$
\ln \frac{1-f+f e^{x}(y+1)}{1+f y}-f x \frac{1+y}{1+f y}=z(x)-z(0)-x z^{\prime}(0)=\int_{0}^{x}(x-u) z^{\prime \prime}(u) d u .
$$

It remains to notice that

$$
0 \leq z^{\prime}(x) \leq 1, \quad 0 \leq z^{\prime \prime}(x) \leq 1,
$$

and then (4.24) follows from (4.26).

With (4.23) and (4.25) in mind, the dominated convergence theorem [9, Theorem IV.32] makes it possible to pass to the limit in probability in (4.22); the convergence in the space $\mathbb{D}$ then follows from the general results of [4, Section IX.5.12]. 
The following is a representation of the long-term growth rate of the limiting wealth process $W^{f}$.

Theorem 4.4. Let $R=R_{t}$ be a Lévy process with representation (4.18). If $\mathbb{E}\left|R_{1}\right|<$ $\infty$, then the process $W^{f}=W_{t}^{f}$ defined in (4.20) satisfies

$$
\begin{aligned}
\lim _{t \rightarrow+\infty} \frac{\ln W_{t}^{f}}{t} & =f\left(\mu+\int_{|x|>1} x F^{R}(d x)\right)+\frac{f(1-f) \sigma^{2}}{2} \\
& +\int_{\mathbb{R}}\left[\ln \left(1+f\left(e^{x}-1\right)\right)-f x\right] F^{R}(d x) .
\end{aligned}
$$

Proof. By (4.20),

$$
\frac{\ln W_{t}^{f}}{t}=f \frac{R_{t}}{t}+\frac{f(1-f) \sigma^{2}}{2}+\frac{1}{t} \int_{0}^{t} \int_{\mathbb{R}}\left[\ln \left(1+f\left(e^{x}-1\right)\right)-f x\right] \mu^{R}(d x, d s) .
$$

It remains to apply the law of large numbers for Lévy processes [10, Theorem 36.5].

If, in addition, we assume that

$$
f_{-1}^{1}|x| F^{R}(d x)<\infty
$$

that is, the small-jump component of $R$ has bounded variation, then, after a change of variables and re-arrangement of terms, (4.27) becomes (3.26). On the other hand, equality (3.26) is derived for a wider class of return processes that includes Lévy processes as a particular case.

Similar to Proposition 2.3, we also have the following result.

Theorem 4.5. In the setting of Theorem 4.4, denote the right-hand side of (4.27) by $g_{R}(f)$ and assume that

$$
\begin{aligned}
& \lim _{f \rightarrow 0+} \int_{\mathbb{R}}\left(\frac{e^{x}-1}{1+f\left(e^{x}-1\right)}-x\right) F^{R}(d x)>-\left(\mu+\frac{\sigma^{2}}{2}+\int_{|x|>1} x F^{R}(d x)\right), \\
& \lim _{f \rightarrow 1-} \int_{\mathbb{R}}\left(\frac{e^{x}-1}{1+f\left(e^{x}-1\right)}-x\right) F^{R}(d x)<-\left(\mu+\int_{|x|>1} x F^{R}(d x)\right),
\end{aligned}
$$

Then there exists a unique $f^{*} \in(0,1)$ such that

$$
g_{R}(f)<g_{R}\left(f^{*}\right)
$$

for all $f$ in the domain of $g_{R}$.

\section{Continuous Limit of Random Discrete Compounding}

The objective of this section is to analyze high frequency limits for betting in business time. In other words, the number of bets is not known a priori, so that a natural 
model of the corresponding wealth process is

$$
W_{t}^{n, f}=\prod_{k=1}^{\left\lfloor\Lambda_{n, t}\right\rfloor}\left(1+f r_{n, k}\right)
$$

where, for each $n$, the process $t \mapsto \Lambda_{n, t}$ is a subordinator, that is, a non-decreasing Lévy process, independent of all $r_{n, k}$.

To study (5.1), we will follow the methodology in [7], where convergence of processes is derived after assuming a suitable convergence of the random variables. The main result in this connection is as follows.

Theorem 5.1. Consider the following objects:

- random variables $X_{n, k}, n, k \geq 1$ such that $\left\{X_{n, k}, k \geq 1\right\}$ are iid for each $n$, with mean zero and, for some $\beta \in[0,1], m_{n}:=\left(\mathbb{E}\left|X_{n, 1}\right|^{\beta}\right)^{1 / \beta}<\infty$;

- random processes $\Lambda_{n}=\Lambda_{n, t}, n \geq 1, t \geq 0$, such that, for each $n, \Lambda_{n}$ is a subordinator independent of $\left\{X_{n, k}, k \geq 1\right\}$ with the properties $\Lambda_{n, 0}=0$, and for some numbers $0<\delta, \delta_{1} \leq 1$ and $C_{n}>0,\left(\mathbb{E} \Lambda_{n, t}^{\delta}\right)^{1 / \delta} \leq C_{n} t^{\delta_{1} / \delta}$.

Assume that there exist infinitely divisible random variables $Y$ and $U$ such that

$$
\lim _{n \rightarrow \infty} \sum_{k=1}^{n} X_{n, k} \stackrel{d}{=} \bar{Y}, \lim _{n \rightarrow \infty} \frac{\Lambda_{n, 1}}{n} \stackrel{d}{=} \bar{U} .
$$

If

$$
\sup _{n}\left(C_{n} m_{n}^{\beta}\right)<\infty
$$

then, as $n \rightarrow \infty$, the sequence of processes

$$
t \mapsto \sum_{k=1}^{\left\lfloor\Lambda_{n, t}\right\rfloor} X_{n, k}, t \in[0, T],
$$

converges, in the Skorokhod topology, to the process $Z=Z_{t}$ such that $Z_{t}=Y_{U_{t}}$, where $Y$ and $U$ are independent Lévy processes satisfying $Y_{1} \stackrel{d}{=} \bar{Y}$ and $U_{1} \stackrel{d}{=} \bar{U}$.

The proof is a word-for-word repetition of the arguments leading to [7, Theorem 1]: the result of [2], together with the assumptions of the theorem, implies

$$
\lim _{n \rightarrow \infty} \sum_{k=1}^{\left\lfloor\Lambda_{n, 1}\right\rfloor} X_{n, k} \stackrel{d}{=} Z_{1}
$$

and therefore the convergence of finite-dimensional distributions for the corresponding processes; together with condition (5.2), this implies the convergence in the Skorokhod space. Because we deal exclusively with Lévy processes, it is possible to avoid the heavy machinery from [4].

We now consider the wealth process (5.1) and apply Theorem 5.1 with

$$
X_{n, k}=\ln \left(1+f r_{n, k}\right)-\mathbb{E} \ln \left(1+f r_{n, k}\right) .
$$


On the one hand, convergence to infinitely divisible distributions other than normal is a very diverse area, with a variety of conditions and conclusions; cf. [1, Chapter XVII, Section 5] or a summary in [6, Section 16.2]. On the other hand, optimal strategy (4.10) seems to persist.

For example, assume that the returns $r_{n, k}$ are as in (4.1), and let $\Lambda_{n, t}=S_{n^{\alpha} t}$, where $\alpha \in(0,1]$ and $S=S_{t}$ is the Lévy process such that $S_{1}$ has the $\alpha$-stable distribution with both scale and skewness parameters equal to 1 . Recall that an $\alpha$-stable Lévy process $L^{\alpha}=L_{t}^{\alpha}$ satisfies the following equality in distribution (as processes):

$$
L_{\gamma t}^{\alpha} \stackrel{\mathcal{L}}{=} \gamma^{1 / \alpha} L_{t}^{\alpha}, \gamma>0
$$

Then

$$
\Lambda_{n, t} \stackrel{\mathcal{L}}{=} n S_{t}
$$

and, in the notations of Theorem 5.1, $\bar{Y}$ is normal with mean zero and variance $\sigma^{2}$. Keeping in mind that

$$
\mathbb{E} \ln \left(1+f r_{n, k}\right)=\mathbb{E} \ln \left(1+f r_{n, 1}\right)=\left(f \mu-\frac{f^{2} \sigma^{2}}{2}\right) n^{-1}+o\left(n^{-1}\right),
$$

we repeat the arguments from [7, Example 1] to conclude that

$$
\lim _{n \rightarrow \infty} \ln W_{t}^{n, f} \stackrel{\mathcal{L}}{=}\left(f \mu-\frac{f^{2} \sigma^{2}}{2}\right) S_{t}+Z_{t},
$$

where $Z_{1}$ has symmetric $2 \alpha$-stable distribution. By (5.3),

$$
S_{t} \stackrel{d}{=} t^{1 / \alpha} S_{1}, \lim _{t \rightarrow+\infty} t^{-1 / \alpha} Z_{t} \stackrel{d}{=} \lim _{t \rightarrow+\infty} t^{-1 /(2 \alpha)} Z_{1} \stackrel{d}{=} 0,
$$

and the "natural" long term growth rate becomes

$$
\lim _{t \rightarrow \infty} t^{-1 / \alpha}\left(\lim _{n \rightarrow \infty} \ln W_{t}^{n, f}\right) \stackrel{d}{=}\left(f \mu-\frac{f^{2} \sigma^{2}}{2}\right) S_{1},
$$

which is random, but, for each realization of $S$, is still maximized by $f^{*}$ from (4.10). Therefore, if the time with which we compound our wealth is random, then the growth rate is also random as we don't know when we will stop compounding, yet it is still maximized by a deterministic fraction. Note that, for the purpose of this computation, the (stochastic) dependence between the processes $S$ and $Z$ is not important.

\section{Conclusions And Further Directions}

The NS-NL condition $f^{*} \in[0,1]$ can fail in many situations. Even in the simple Bernoulli model, if $p<1 / 2$, then the short position $f^{*}=2 p-1$ achieves positive long-time wealth growth:

$$
g_{r}\left(f^{*}\right)=p \ln \frac{p}{1-p}+(2-p) \ln (2-2 p)=\ln 2+p \ln p+(1-p) \ln (1-p)>0
$$

Note that $-p \ln p-(1-p) \ln (1-p)$ is the Shannon entropy of the Bernoulli distribution, and the largest value of the entropy is $\ln 2$, corresponding to $p=1 / 2$. When the edge is too big (cf. (2.14) ), then $f^{*}>1$, that is, leveraged gambling leads to bigger long-time wealth growth than any NS-NL strategy. The economical and financial implications of $f^{*} \notin[0,1]$ are beyond the scope of our investigation and must be studied in a 
broader context of risk tolerance: even when $f^{*} \in(0,1)$, a certain fraction of $f^{*}$ can be a smarter strategy, cf. [12, Section 7.3].

A related observation, to be further studied in the future, is that high-frequency betting can lead to a more aggressive strategy than the "low frequency" counterpart. For example, comparing (1.1) and (4.12), we see that $\mu=2 p-1$ and $\sigma^{2}=4 p(1-p)<1$ when $p \neq 1 / 2$. As a result, by (4.10), the optimal strategy for (4.12) with large $n$ is $f^{*} \approx(2 p-1) /(4 p(1-p))>2 p-1$; recall that $f^{*}=2 p-1$ is the optimal strategy for the simple Bernoulli model (1.1). On the other hand, numerical simulations suggest that, in the log-normal model (4.14), (4.15), high-frequency compounding does not always lead to larger $f^{*}$.

Other problems warranting further investigation include

(1) A dynamic strategy $f=f(t)$ with a predictable process $f$;

(2) A portfolio of bets, with a vector of strategies $\mathbf{f}=\left(f_{1}, \ldots, f_{N}\right)$.

\section{REFERENCES}

1. W. Feller, An Introduction to Probability Theory and its Applications, Vol. II, Second edition, John Wiley \& Sons, Inc., 1971.

2. B. V. Gnedenko, G. Fahim, A certain transfer theorem. Dokl. Akad. Nauk SSSR, 187, pp. 15-17, 1969.

3. I. S. Gradshteyn, I. M. Ryzhik, Table of Integrals, Series, and Products, Eighth edition, Elsevier/Academic Press, 2015.

4. J. Jacod, A. N. Shiryaev, Limit Theorems for Stochastic Processes, Second edition, Springer, 2003.

5. J. L. Kelly, A new interpretation of information rate, Bell. System Tech. J., 1956.

6. A. Klenke, Probability Theory: A Comprehensive Course, Second edition, Universitext, Springer, 2014.

7. V. Yu. Korolev, L. M. Zaks, A. I. Zeifman, On convergence of random walks generated by compound Cox processes to Lévy processes, Statist. Probab. Lett., 83(10), pp. 2432-2438, 2013.

8. R. Sh. Liptser, A. N. Shiryayev, Theory of Martingales, Kluwer, 1989.

9. P. Protter, Stochastic Integration and Differential Equations, Springer, 1990.

10. K. Sato, Lévy Processes and Infinitely Divisible Distributions, Cambridge University, 1999.

11. E. O. Thorp, Understanding the Kelly criterion, in: L.C. MacLean, W. T. Ziemba, and E. O. Thorp (editors), The Kelly Capital Growth Investment Criterion, World Scientific, 2010.

12. E. O. Thorp, The Kelly criterion in blackjack, sports betting, and the stock market, in: L.C. MacLean, W. T. Ziemba, and E. O. Thorp (editors), The Kelly Capital Growth Investment Criterion, World Scientific, 2010.

13. E. O. Thorp, A Perspective on Quantitative Finance: Models for Beating the Market, in: The Best of Wilmott, 2003.

14. V. M. Zolotarev, Asymptotic expansions with probability 1 of sums of independent random variables, Teor. Veroyatnost. i Primenen., 36(4), pp. 770-772, 1991.

Sergey Lototsky, Department of Mathematics, USC, Los Angeles, CA 90089, USA

E-mail address: lototsky@usc.edu

Austin Pollok, Department of Mathematics, USC, los Angeles, CA 90089, USA

E-mail address: pollok@usc.edu 